\title{
Activation of Erk and p53 regulates copper oxide nanoparticle-induced cytotoxicity in keratinocytes and fibroblasts
}

This article was published in the following Dove Press journal:

International Journal of Nanomedicine

10 October 2014

Number of times this article has been viewed

\author{
Cheng Luo' \\ Yan $\mathrm{Li}^{2}$ \\ Liang Yang' \\ Yan Zheng ${ }^{3}$ \\ Jiangang Long' \\ Jinjing Jia ${ }^{3}$ \\ Shengxiang $\mathrm{Xiao}^{3}$ \\ Jiankang Liu' \\ 'Center for Mitochondrial Biology \\ and Medicine, The Key Laboratory \\ of Biomedical Information \\ Engineering of Ministry \\ of Education, School of Life Science \\ and Technology and Frontier \\ Institute of Science and Technology, \\ ${ }^{2}$ Center for Bioinformatics, The Key \\ Laboratory of Biomedical Information \\ Engineering of Ministry of Education, \\ School of Life Science and Technology, \\ Xi'an Jiaotong University, Xi'an, \\ People's Republic of China; \\ ${ }^{3}$ Department of Dermatology, \\ The 2nd Affiliated Hospital of Xi'an \\ Jiaotong University, Xi'an, People's \\ Republic of China
}

Correspondence: Yan Zheng

Department of Dermatology, The 2nd Affiliated Hospital of Xi'an Jiaotong University, I 57 XiWu Road, Xi'an, 710004, People's Republic of China Tel +862987679969

Fax +862982665849

Email zenyan66@I26.com

Jiankang Liu

Center for Mitochondrial Biology and Medicine, X'an Jiaotong University

School of Life Science and Technology,

Xi'an 710049, People's Republic of China

Tel +862982665429

Fax +862982665849

Email j.liu@mail.xjtu.edu.cn
Abstract: Copper oxide nanoparticles (CuONP) have attracted increasing attention due to their unique properties and have been extensively utilized in industrial and commercial applications. For example, their antimicrobial capability endows CuONP with applications in dressings and textiles against bacterial infections. Along with the wide applications, concerns about the possible effects of CuONP on humans are also increasing. It is crucial to evaluate the safety and impact of $\mathrm{CuONP}$ on humans, and especially the skin, prior to their practical application. The potential toxicity of CuONP to skin keratinocytes has been reported recently. However, the underlying mechanism of toxicity in skin cells has remained unclear. In the present work, we explored the possible mechanism of the cytotoxicity of CuONP in HaCaT human keratinocytes and mouse embryonic fibroblasts (MEF). CuONP exposure induced viability loss, migration inhibition, and $\mathrm{G}_{2} / \mathrm{M}$ phase cycle arrest in both cell types. CuONP significantly induced mitogen-activated protein kinase (extracellular signal-regulated kinase [Erk], p38, and c-Jun N-terminal kinase [JNK]) activation in dose- and time-dependent manners. U0126 (an inhibitor of Erk), but not SB 239063 (an inhibitor of p38) or SP600125 (an inhibitor of JNK), enhanced CuONP-induced viability loss. CuONP also induced decreases in p53 and p-p53 levels in both cell types. Cyclic pifithrin- $\alpha$, an inhibitor of 553 transcriptional activity, enhanced CuONP-induced viability loss. Nutlin-3 $\alpha$, a p53 stabilizer, prevented $\mathrm{CuONP}$-induced viability loss in $\mathrm{HaCaT}$ cells, but not in MEF cells, due to the inherent toxicity of nutlin-3 $\alpha$ to MEF. Moreover, the experiments on primary keratinocytes are in accordance with the conclusions acquired from $\mathrm{HaCaT}$ and MEF cells. These data demonstrate that the activation of Erk and p53 plays an important role in CuONP-induced cytotoxicity, and agents that preserve Erk or p53 activation may prevent CuONP-induced cytotoxicity.

Keywords: cell cycle arrest, CuONP, MAPK, nutlin-3 $\alpha$, cyclic pifithrin- $\alpha$

\section{Introduction}

Copper oxide nanoparticles (CuONP) have been widely applied in semiconductors, catalysts, microelectronic materials, and lithium batteries. ${ }^{1}$ Similar to silver and $\mathrm{ZnO}$ nanoparticles, CuONP exhibit excellent antimicrobial efficiency. ${ }^{2-4}$ Much attention has been paid to the feasible applications of CuONP, such as antimicrobial masks ${ }^{5}$ and textiles. ${ }^{6,7}$ However, it is crucial to evaluate their potential effects on the environment and human health before their practical application. Certain reports have revealed that $\mathrm{CuONP}$ are toxic to the aquatic macrophyte Lemna gibba ${ }^{8}$ and Xenopus laevis. ${ }^{9}$ CuONP were also reported to be cytotoxic to human cells, such as brain, ${ }^{10}$ lung, ${ }^{11,12}$ liver, ${ }^{13,14}$ kidney, ${ }^{15}$ and skin ${ }^{16}$ cells. A test using the human lung adenocarcinoma cell line A549 implied that CuONP were more toxic than other metal oxide nanoparticles $\left(\mathrm{TiO}_{2}, \mathrm{CuZnFe}_{2} \mathrm{O}_{4}, \mathrm{Fe}_{3} \mathrm{O}_{4}\right.$, and $\mathrm{Fe}_{2} \mathrm{O}_{3}$ ) and carbon-based nanomaterials (carbon nanopowders and multiwalled carbon nanotubes).${ }^{17}$ Organs such as the lung and skin were 
more vulnerable to nanoparticles due to their direct contact with the external environment. Many studies have focused on CuONP-induced cytotoxicity and corresponding mechanisms in human lung epithelial cells. ${ }^{11,12,17-19}$ Oxidative stress and autophagy have been reported to play vital roles in CuONPinduced cytotoxicity. ${ }^{12,18}$ Hanagata et al reported that $\mathrm{CuONP}$ exposure upregulated genes involved in mitogen-activated protein kinase (MAPK) pathways while downregulating genes involved in cell cycle progression. ${ }^{11}$ Semisch et al showed that compared with microscale $\mathrm{CuO}$ particles and copper chloride, $\mathrm{CuONP}$ were more cytotoxic and induced genotoxicity due to their nanoscale features..$^{20} \mathrm{CuONP}$ were also reported to induce oxidative stress and apoptosis in $\mathrm{HaCaT}$ human keratinocytes. However, the decisive factors and the accompanying changes involved in the toxicity of $\mathrm{CuONP}$ are unclear. ${ }^{16}$ Moreover, the underlying mechanism of the toxicity of CuONP to skin-associated cells has not been clarified. In the present work, we examined the cytotoxicity
A

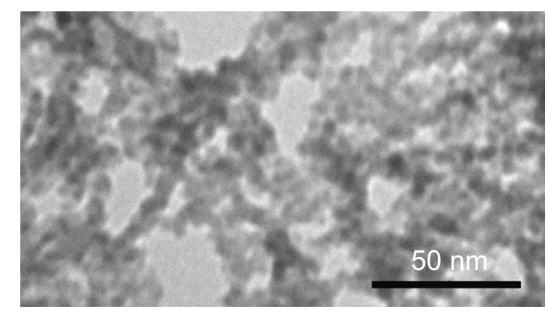

B

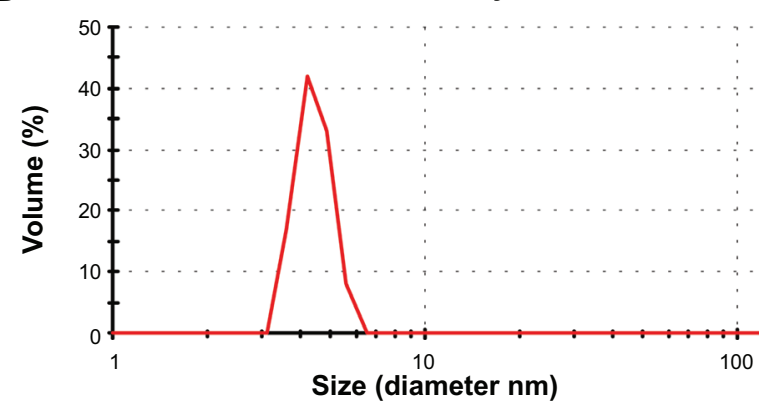

C

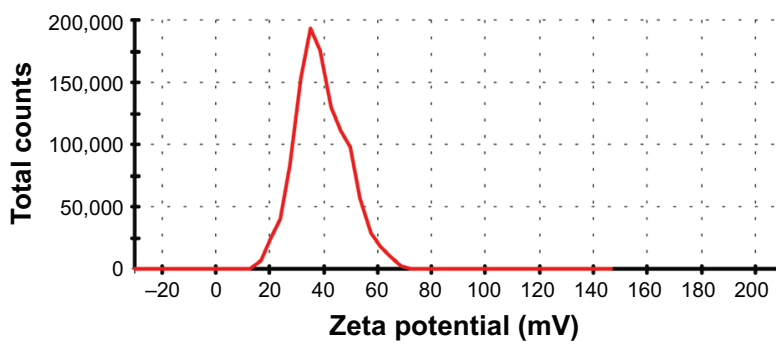

of CuONP to skin-associated cells, keratinocytes (HaCaT) and mouse embryonic fibroblasts (MEF), and investigated the underlying molecular mechanisms.

\section{Materials and methods \\ Materials}

CuONP was synthesized as $\mathrm{Zhu}$ et al reported, ${ }^{21}$ and characterized using transmission electron microscope H-7650 (Hitachi Ltd., Tokyo, Japan), a Malvern Zetasizer Nano ZS90 (Malvern Instruments, Malvern, UK), and X-ray Photoelectron Spectrometer (Thermo Fisher Scientific, Waltham, MA, USA) (Figure 1). 3-(4,5-dimethyl-2-thiazolyl)2,5-diphenyltetrazolium bromide (MTT), SB 239063, U0126, propidium iodide, SP600125, and anti-actin primary antibody were purchased from Sigma-Aldrich Co. (St Louis, MO, USA). Fetal bovine serum (FBS) was purchased from Biological Industries Israel Beit-Haemek Ltd. (Kibbutz Beit-Haemek, Israel). Dulbecco's Modified Eagle's Medium (DMEM),
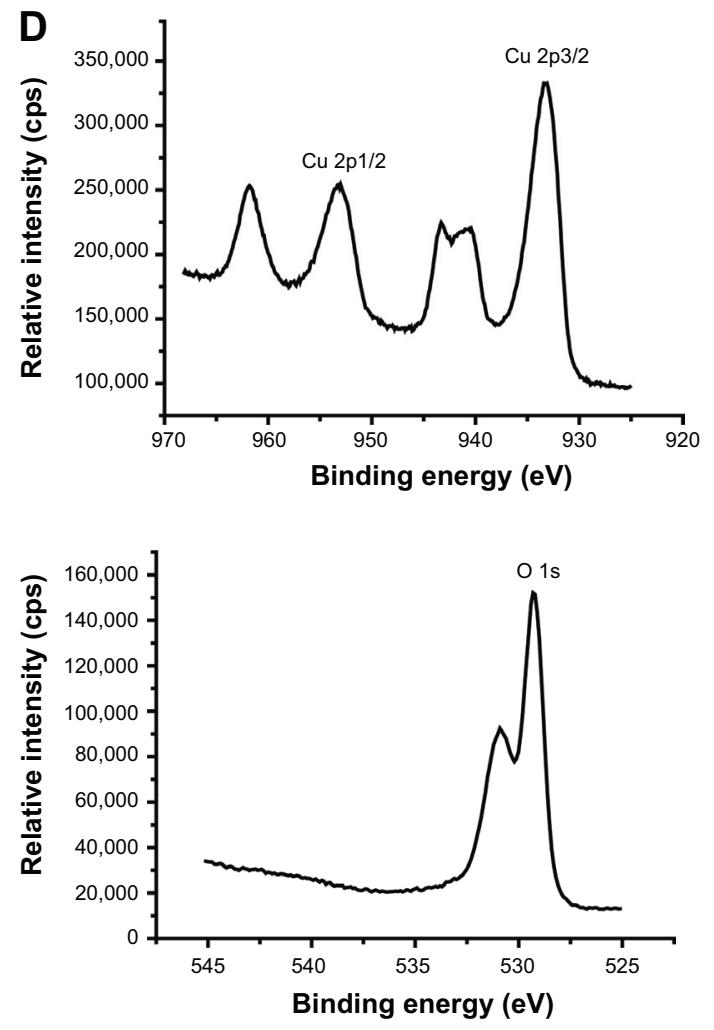

Figure I Physicochemical characterization of the CuONP.

Notes: (A) Transmission electron microscope image. (B) Hydrodynamic diameter. (C) Zeta potential. (D) Chemical composition by X-ray photoelectron spectrometer analysis.

Abbreviations: CuONP, copper oxide nanoparticles; cps, counts per second. 
penicillin, streptomycin, and trypsin were purchased from Thermo Fisher Scientific. Primary antibodies against p-p53 and phosphorylated extracellular signal-regulated kinase (Erk) were obtained from Cell Signaling Technology, Inc. (Beverly, MA, USA). Primary antibodies against cyclin A, cyclin B1, p53, phosphorylated c-Jun N-terminal kinase (JNK), JNK, p-p38, p38, Erk, and cyclic pifithrin- $\alpha$ (PFT- $\alpha)$ were purchased from Santa Cruz Biotechnology Inc. (Dallas, TX, USA). Horseradish peroxidase-conjugated secondary antibodies were purchased from Jackson ImmunoResearch Laboratories, Inc. (West Grove, PA, USA). The bicinchoninic acid assay kit and enhanced chemiluminescence Western blotting substrate were obtained from Thermo Fisher Scientific. Cell lysis buffer was purchased from Beyotime Biotechnology (Haimen, Jiangsu, People's Republic of China). Other chemicals were obtained from local suppliers. Deionized water was used in all experiments.

\section{Cell culture and treatment}

Human keratinocytes $\mathrm{HaCaT}$ and mouse embryonic fibroblast cells were cultured in DMEM supplemented with 10\% FBS and penicillin $(100 \mathrm{U} / \mathrm{mL}) /$ streptomycin $(100 \mu \mathrm{g} / \mathrm{mL})$ in a $5 \% \mathrm{CO}_{2}$ humidified incubator at $37^{\circ} \mathrm{C}$ (Thermo Fisher Scientific). In a dose-dependent manner, $\mathrm{HaCaT}$ and MEF cells were treated with the indicated concentrations of $\mathrm{CuONP}$ for 24 hours. In a time-dependent manner, HaCaT or MEF cells were treated with 60 or $80 \mu \mathrm{mol} / \mathrm{L} \mathrm{CuONP}$, respectively.

For the MAPK-inhibitor treatments, $\mathrm{HaCaT}$ cells were pretreated with $5 \mu \mathrm{mol} / \mathrm{L} \mathrm{U} 0126$ for 2 hours and then treated with 20 or $40 \mu \mathrm{g} / \mathrm{mL} \mathrm{CuONP}$ for 24 hours; HaCaT cells were pretreated with $20 \mu \mathrm{mol} / \mathrm{L} \mathrm{SB} 239063$ or $20 \mu \mathrm{mol} / \mathrm{L} \mathrm{SP} 600125$ for 2 hours and then treated with 20 or $60 \mu \mathrm{g} / \mathrm{mL} \mathrm{CuONP}$ for 24 hours; MEF cells were pretreated with $5 \mu \mathrm{mol} / \mathrm{L}$ U0126 for 2 hours and then treated with 40 or $60 \mu \mathrm{g} / \mathrm{mL}$ CuONP for 24 hours; MEF cells were pretreated with $20 \mu \mathrm{mol} / \mathrm{L} \mathrm{SB}$ 239063 or $20 \mu \mathrm{mol} / \mathrm{L} \mathrm{SP} 600125$ for 2 hours and then treated with 40 or $80 \mu \mathrm{g} / \mathrm{mL}$ CuONP for 24 hours.

For the p53-transcriptional-inhibitor (cyclic PFT- $\alpha$ ) treatment, $\mathrm{HaCaT}$ cells were pretreated with 1 or $10 \mu \mathrm{mol} / \mathrm{L}$ cyclic PFT- $\alpha$ for 2 hours and then treated with $20 \mu \mathrm{g} / \mathrm{mL}$ CuONP for 24 hours or with $60 \mu \mathrm{g} / \mathrm{mL}$ CuONP for 6 hours, respectively. MEF cells were pretreated with 1 or $10 \mu \mathrm{mol} / \mathrm{L}$ cyclic PFT- $\alpha$ for 2 hours and then treated with $40 \mu \mathrm{g} / \mathrm{mL}$ CuONP for 24 hours or with $80 \mu \mathrm{g} / \mathrm{mL}$ CuONP for 3 hours, respectively. For p53-stabilizer (nutlin-3 $\alpha$ ) treatment, $\mathrm{HaCaT}$ or MEF cells were pretreated with $30 \mu \mathrm{mol} / \mathrm{L}$ nutlin-3 $\alpha$ for 2 hours and then treated with $60 \mu \mathrm{g} / \mathrm{mL}$ or $80 \mu \mathrm{g} / \mathrm{mL} \mathrm{CuONP}$ for 24 hours, respectively.

\section{Cellular viability assessment}

The cells were washed with FBS-free DMEM once and incubated with $0.5 \mathrm{mg} / \mathrm{mL}$ MTT in FBS-free DMEM for 1 hour at $37^{\circ} \mathrm{C}$ in a $\mathrm{CO}_{2}$ incubator. The formazan generated was dissolved in DMSO and recorded at $490 \mathrm{~nm}$ with a microplate reader (Molecular Devices LLC, Sunnyvale, CA, USA). ${ }^{22}$

\section{Migratory assay}

The cells were scratched with a $200 \mu \mathrm{L}$ pipette tip across the center of a plate to create a wound. The detached debris was washed off with FBS-free DMEM. The cells were treated with the indicated concentrations of CuONP. Photographs were taken at 0 and 24 hours after the treatment. ${ }^{23}$

\section{Cell cycle analysis}

The cells were trypsinized and washed with PBS once. The cells were then resuspended in PBS, followed by fixation in ice-cold $70 \%$ ethanol and storage at $-20^{\circ} \mathrm{C}$ for at least 24 hours. The fixed cells were collected by centrifugation at 1,000 rpm for 10 minutes and washed with PBS once, followed by incubation with a propidium iodide working solution $(0.1 \%$ Triton- $100,200 \mu \mathrm{g} / \mathrm{mL}$ RNase A, and $50 \mu \mathrm{g} / \mathrm{mL}$ stock solution) for 15 minutes in the dark and analysis by flow cytometry. ${ }^{24}$

\section{Western blot analysis}

The cells were lysed and centrifuged at 13,000 rpm for 15 minutes at $4{ }^{\circ} \mathrm{C}$ to collect the supernatant. The protein content in the lysate's supernatant was quantified with a bicinchoninic acid assay kit. The proteins were separated by sodium dodecyl sulfate polyacrylamide gel electrophoresis and transferred to nitrocellulose membranes. The membranes were blocked with 5\% non-fat milk in Tris-buffered saline containing $0.1 \%$ Tween 20 (TBST) for 1 hour at room temperature, probed with primary antibodies against the target proteins overnight at $4^{\circ} \mathrm{C}$, and then washed with TBST to remove unbounded antibodies. Next, the membranes were incubated with horseradish peroxidase-conjugated secondary antibodies for 1 hour at room temperature and washed with TBST again. Specific signals on the probed membranes were detected using enhanced chemiluminescence and an X-ray-film exposure system.

\section{Statistical analysis}

A one-way analysis of variance, followed by Fisher's least significant difference test, was used to evaluate the significant differences among the treatments. The data are presented as the mean \pm standard deviation $(\mathrm{n}>3)$. Statistical significance was indicated as follows: $* P<0.05$ and $* * P<0.01$. 


\section{Results}

\section{CuONP induce cytotoxicity in $\mathrm{HaCaT}$ and MEF cells}

CuONP significantly suppressed the proliferation of $\mathrm{HaCaT}$ and MEF cells in a dose-dependent manner in 24 hours' treatment (Figure 2A and B). Cellular migration capacities were also inhibited in both CuONP-treated cells in scratch-wound assay (Figure $2 \mathrm{C}$ and $\mathrm{D}$ ).

Cell cycle arrest may promote growth and migration inhibition in CuONP-treated $\mathrm{HaCaT}$ and MEF cells. To verify this assumption, CuONP-treated cells were subjected to cell cycle analysis with flow cytometry. There was a significant increase in the population of cells in $\mathrm{G}_{2} / \mathrm{M}$ phase (from $3.9 \%$ to $42.8 \%$ in $\mathrm{HaCaT}$, or from $1.2 \%$ to $32.8 \%$ in MEF), with corresponding decreases in the $\mathrm{G}_{1}$ and $\mathrm{S}$ phases after 24 hours' CuONP treatment, which implied that the cell cycle was arrested in $\mathrm{G}_{2} / \mathrm{M}$ phase in a dose-dependent manner in CuONP-treated cells (Figure $3 \mathrm{~A}$ and B). There was a doseand time-dependent decrease in the levels of cyclin $\mathrm{A}$ and cyclin $\mathrm{B} 1$ in CuONP-treated $\mathrm{HaCaT}$ cells (Figure $3 \mathrm{C}$ and D) and MEF cells (Figure $3 \mathrm{E}$ and $\mathrm{F}$ ). The downregulation of those $\mathrm{G}_{2} / \mathrm{M}$ transition-related proteins further revealed that $\mathrm{CuONP}$ induced $\mathrm{G}_{2} / \mathrm{M}$ phase arrest.

A

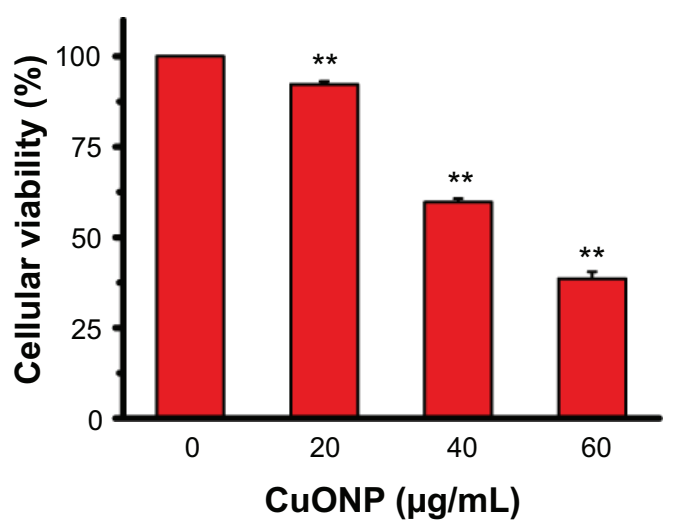

C

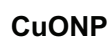
$(\mu \mathrm{g} / \mathrm{mL})$

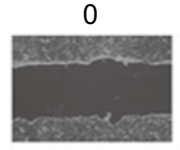

0

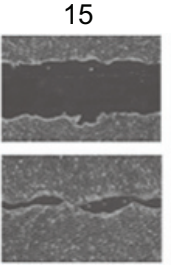

20

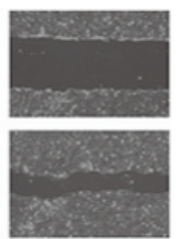

Erk activation may prevent

CuONP-induced growth inhibition

in $\mathrm{HaCaT}$ and MEF cells

The activation of Erk, p38, and JNK was significantly induced in dose- and time-dependent manners in CuONPtreated $\mathrm{HaCaT}$ cells (Figure 4A and B) and MEF cells (Figure $4 \mathrm{C}$ and D). The inhibition of Erk activation by pretreatment with U0126 significantly promoted growth inhibition relative to treatment with $\mathrm{CuONP}$ alone in $\mathrm{HaCaT}$ cells (Figure 4E) and MEF cells (Figure 4F). However, pretreatment with SB 239063 (an inhibitor of p38 activation) and SP600125 (an inhibitor of JNK activation) exhibited little or no effect on the viabilities of CuONP-treated HaCaT cells (Figure 4G) and MEF cells (Figure 4H). These data implied that Erk plays a vital role in CuONP-induced cytotoxicity, whereas the changes in p38 and JNK were incidental.

\section{Loss of p53 accelerates CuONP-induced growth inhibition in $\mathrm{HaCaT}$ and MEF cells}

There was a remarkable decrease in the protein levels of p53 and p-p53 in a dose-dependent manner in CuONPtreated $\mathrm{HaCaT}$ cells (Figure 5A) and MEF cells (Figure 5B).

B

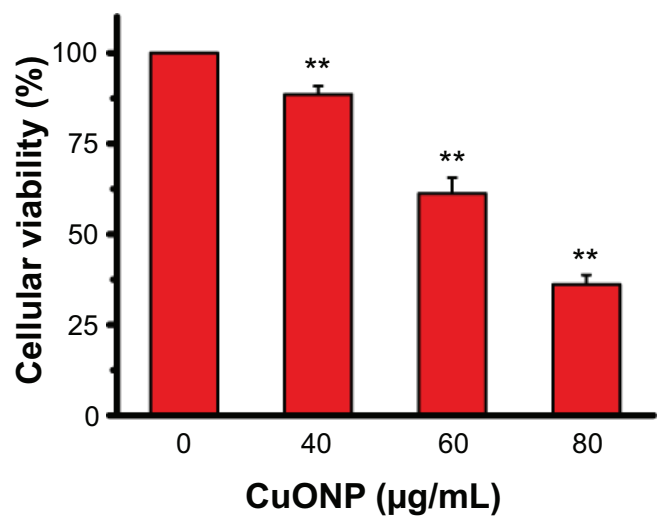

D

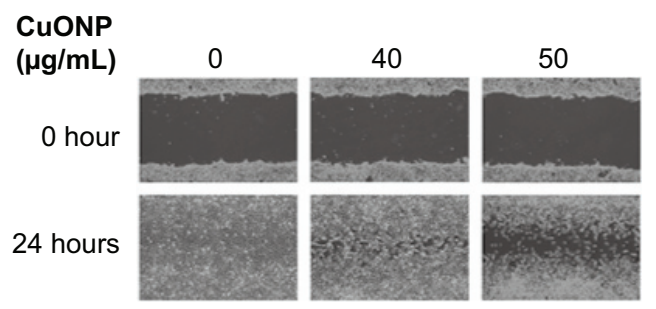

Figure $2 \mathrm{CuONP}$-induced cytotoxicity in $\mathrm{HaCaT}$ and MEF cells.

Notes: Viability loss in HaCaT (A) and MEF (B) cells with CuONP treatment for 24 hours. Migration inhibition in CuONP-treated HaCaT (C) and MEF (D) cells. The data are presented as the mean \pm standard deviation $(n>3)$. ${ }^{*} * P<0.01$ versus vehicle control.

Abbreviations: CuONP, copper oxide nanoparticles; MEF, mouse embryonic fibroblasts. 
A

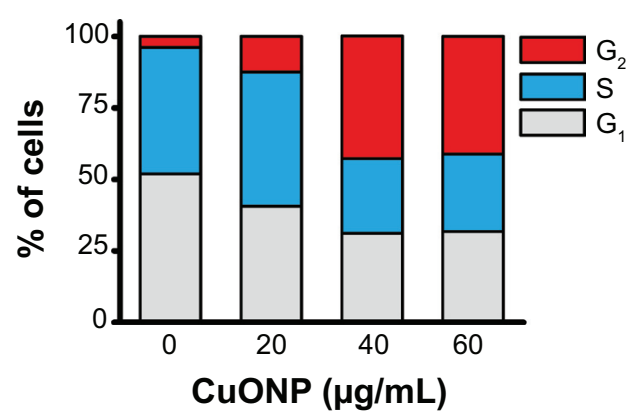

C

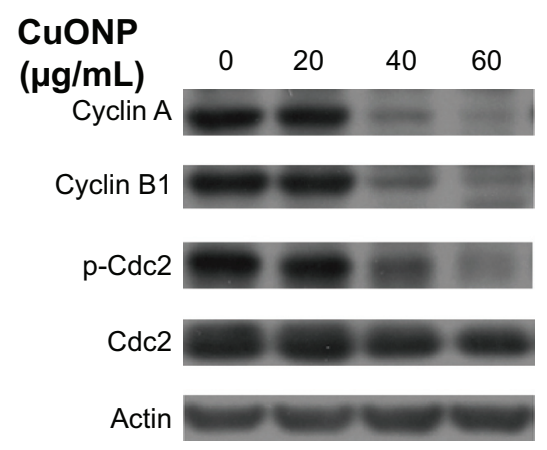

E

CuO

$(\mu \mathrm{g} / \mathrm{mL})$

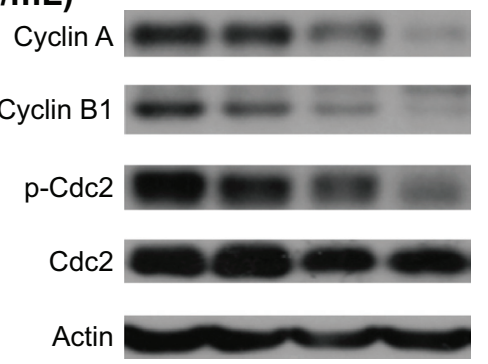

B

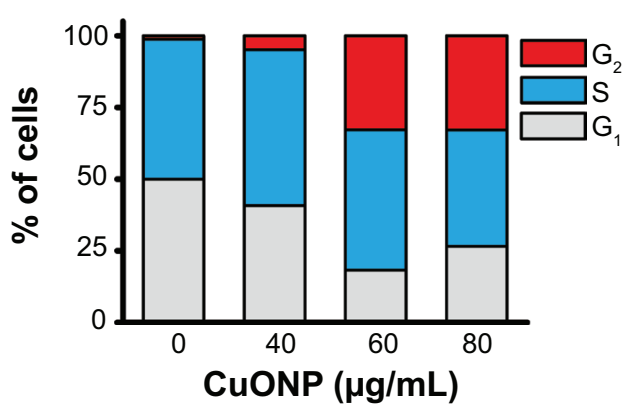

D

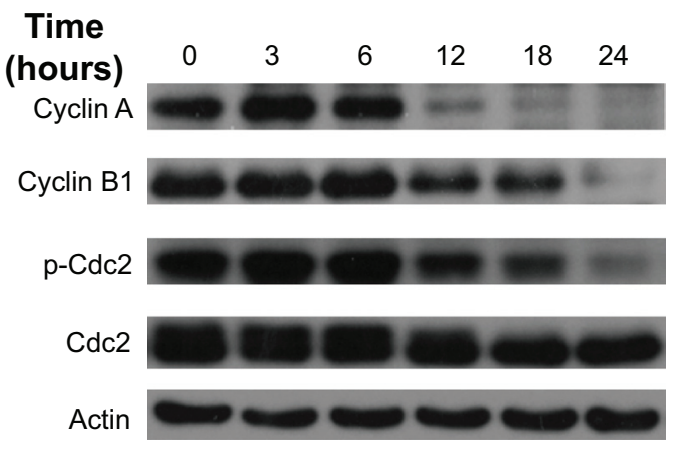

$\mathbf{F}$

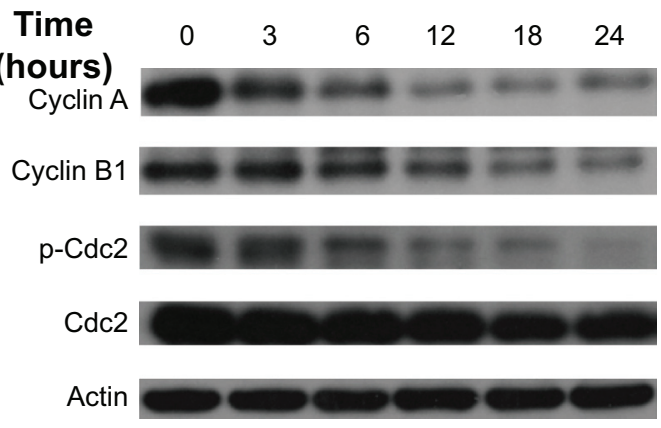

Figure $3 \mathrm{CuONP}$-induced cell cycle arrest in $\mathrm{HaCaT}$ and MEF cells.

Notes: Cell cycle analysis by flow cytometry for CuONP-treated $\mathrm{HaCaT}(\mathbf{A})$ and MEF (B) cells. Western blot analysis of the proteins associated with the $\mathrm{G}_{2} / \mathrm{M}$ transition in CuONP-treated $\mathrm{HaCaT}(\mathbf{C}, \mathbf{D})$ and $\operatorname{MEF}(\mathbf{E}, \mathbf{F})$ cells.

Abbreviations: CuONP, copper oxide nanoparticles; MEF, mouse embryonic fibroblasts.

Pretreatment with cyclic PFT- $\alpha$, a p53 transcriptional inhibitor, promoted growth inhibition relative to treatment with CuONP alone in $\mathrm{HaCaT}$ cells (Figure 5C and D) and MEF cells (Figure 5E and F). Briefly, pretreatment with $1 \mu \mathrm{mol} / \mathrm{L}$ cyclic PFT- $\alpha$ promoted growth inhibition in both cells with low doses of CuONP for 24 hours' treatment (Figure $5 \mathrm{C}$ and $\mathrm{E}$ ), and pretreatment with $10 \mu \mathrm{mol} / \mathrm{L}$ cyclic PFT- $\alpha$ promoted growth inhibition in high doses of $\mathrm{CuONP}$ for 6 hours' (for HaCaT) or 3 hours' (for MEF) treatment (Figure 5D and F). Moreover, pretreatment with nutlin-3 $\alpha$, a murine double minute-2 (MDM2)/p53 inhibitor that stabilizes p53, prevented high dose of CuONP-induced viability loss in HaCaT cells (Figure 5G). Nutlin-3 $\alpha$ itself was toxic to MEF cells, so no inhibition of the viability loss induced by CuONP was observed (Figure $5 \mathrm{H}$ ).

\section{CuONP-induced cytotoxicity in primary keratinocytes}

Primary rat keratinocytes were isolated from newborn rat skins according to a report from Albarenque and Doi. ${ }^{25} \mathrm{CuONP}$ induced dose-dependent viability loss in primary keratinocytes (Figure 6A). Erk inhibitor (U0126) and p53 transcriptional inhibitor (cyclic PFT- $\alpha$ ) promoted CuONP-induced viability loss (Figure 6B). Nutlin-3 $\alpha$ was toxic to primary keratinocytes, which is the same as the MEF; no inhibition of the viability loss induced by CuONP was observed. 
A

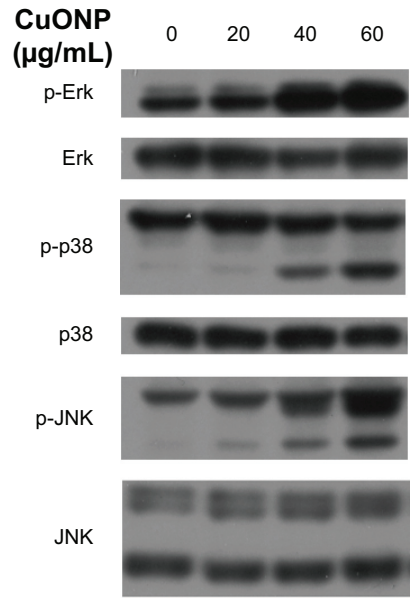

C

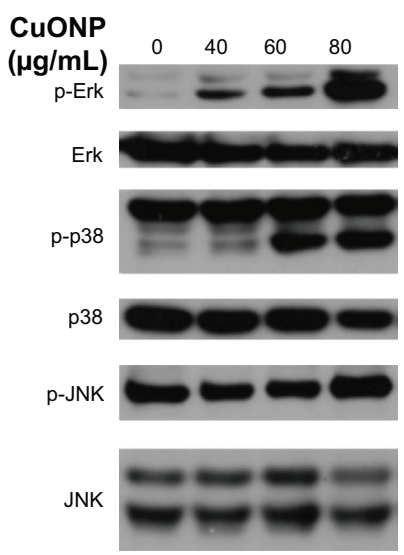

E

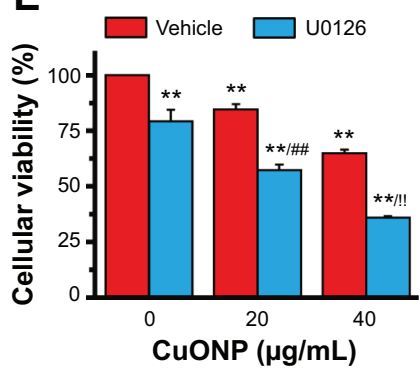

G

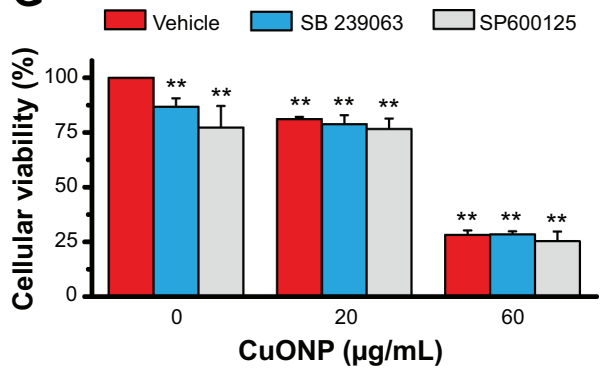

B

D

Time $\begin{array}{lllllll}\text { Time } & 0 & 3 & 6 & 12 & 18 & 24\end{array}$

(hours)

p-Erk

Erk

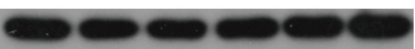

p-p38

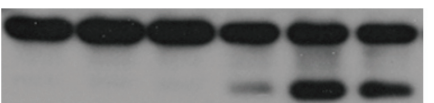

p38

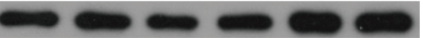

p-JNK

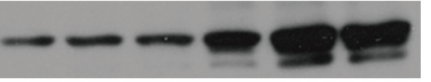

JNK

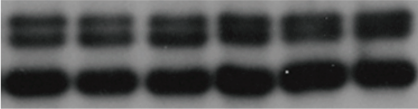

$\begin{array}{lllllll}\text { (hours) } & 0 & 3 & 6 & 12 & 18 & 24\end{array}$

p-Erk

Erk

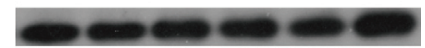

p-p38

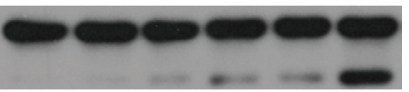

p38

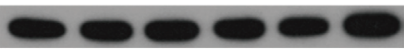

p-JNK

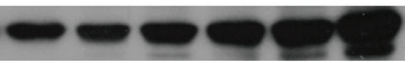

JNK
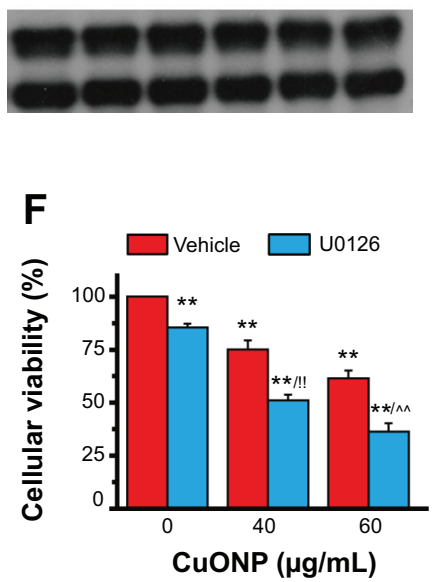

H

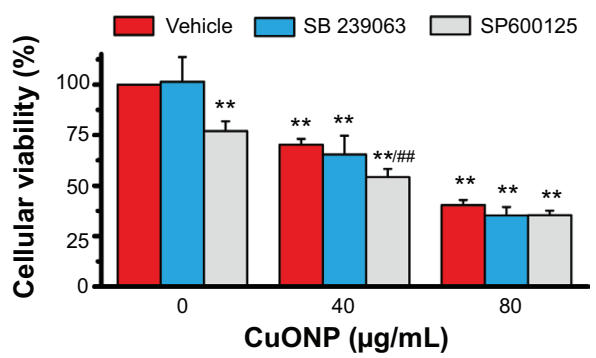

Figure 4 Erk was involved in CuONP-induced cytotoxicity in $\mathrm{HaCaT}$ and MEF cells.

Notes: Western blot analysis of CuONP dose-dependent MAPK activation in HaCaT (A) and MEF (C) cells; Western blot analysis of CuONP time-dependent MAPK activation in $60 \mu \mathrm{g} / \mathrm{mL}$ CuONP-treated HaCaT (B) and $80 \mu \mathrm{g} / \mathrm{mL}$ CuONP-treated MEF (D) cells; the effects of MAPK inhibitors (U0I26, SP600I25, and SB 239063) on CuONP-induced viability loss in $\mathrm{HaCaT}(\mathbf{E}, \mathbf{G})$ and $\mathrm{MEF}(\mathbf{F}, \mathbf{H})$ cells. The data are presented as the mean \pm standard deviation $(\mathrm{n}>3)$. $* * P<0.0 \mathrm{I}$ versus vehicle control. ${ }^{\#} P<0.0$ I versus $20 \mu \mathrm{g} / \mathrm{mL}$ CuONP group. " $P<0.0$ I versus $40 \mu \mathrm{g} / \mathrm{mL}$ CuONP group. ${ }^{\wedge} P<0.01$ versus $60 \mu \mathrm{g} / \mathrm{mL}$ CuONP group.

Abbreviations: CuONP, copper oxide nanoparticles; MEF, mouse embryonic fibroblasts; MAPK, mitogen-activated protein kinase. 
A

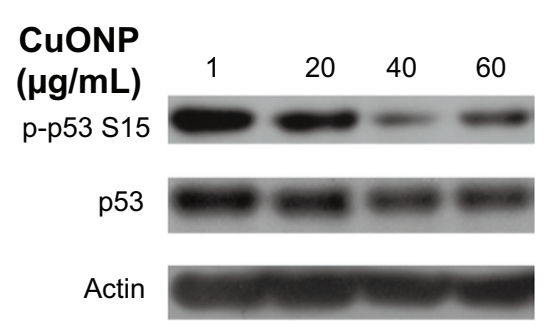

C

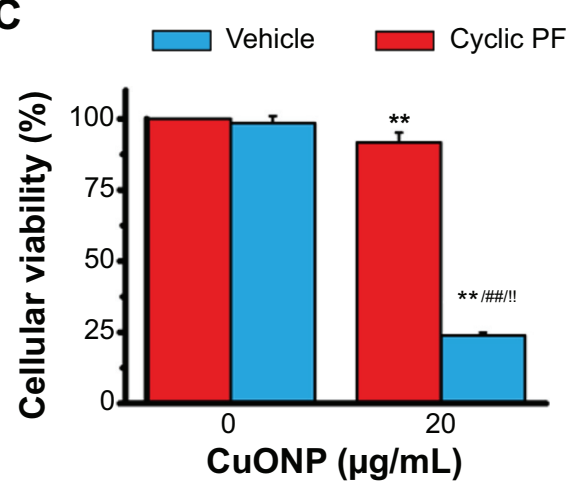

E

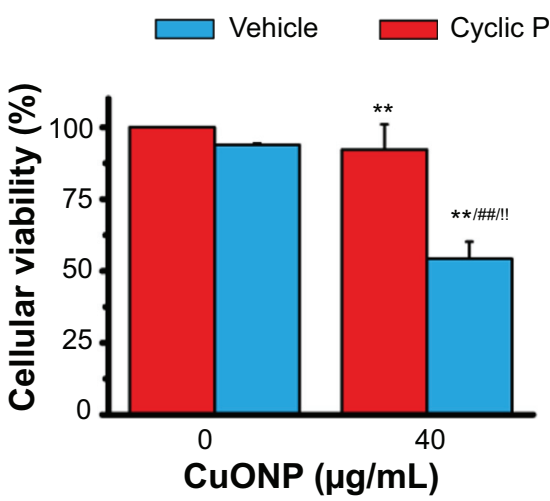

G

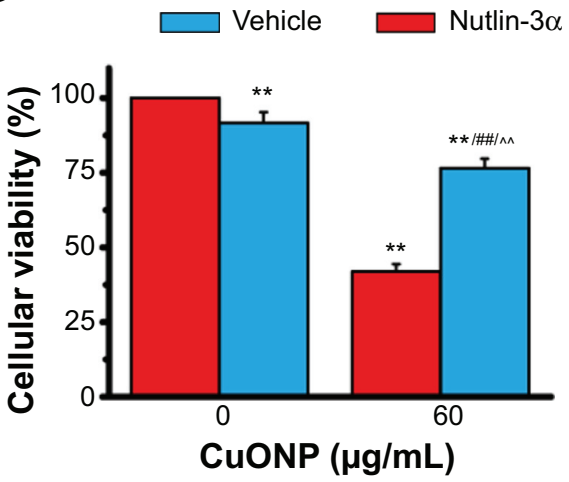

B

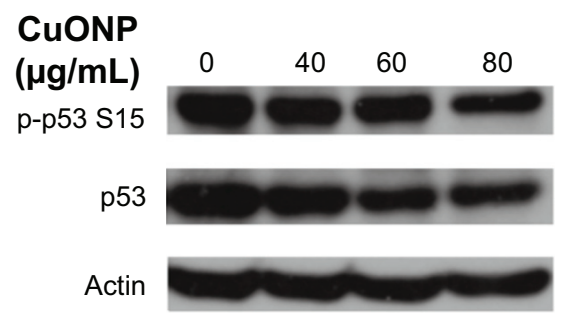

D

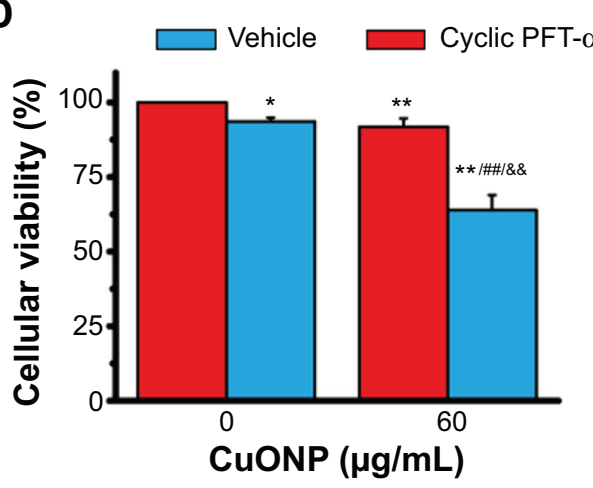

$\mathbf{F}$

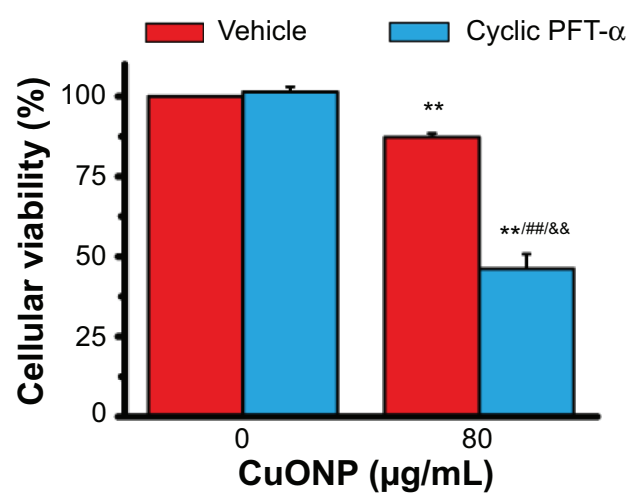

H

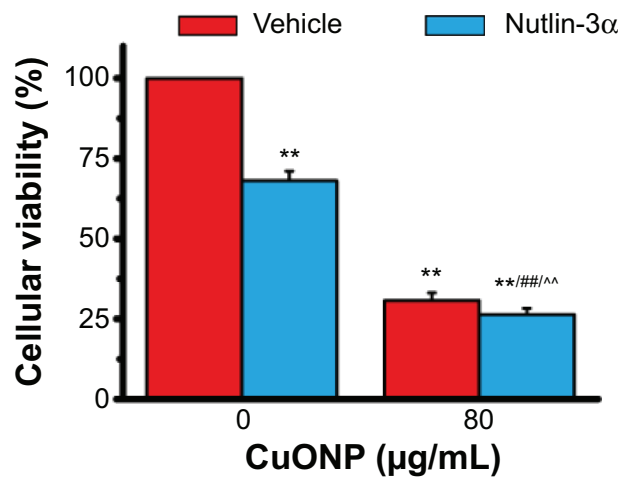

Figure 5 p53 was involved in CuONP-induced cytotoxicity in $\mathrm{HaCaT}$ and MEF cells.

Notes: Western blot analysis of $\mathrm{p} 53$ and $\mathrm{p}-\mathrm{p} 53$ in CuONP-treated HaCaT (A) and MEF (B) cells. The effects of I $\mu$ mol/L p53 transcriptional inhibitor (cyclic PFT- $\alpha$ ) on $\mathrm{CuONP}$-induced viability loss in $\mathrm{HaCaT}$ (C) and MEF (E) cells with CuONP treatment for 24 hours. The effects of $10 \mu \mathrm{mol} / \mathrm{L}$ cyclic PFT- $\alpha$ on CuONP-induced viability loss in $\mathrm{HaCaT}$ cells with CuONP treatment for 6 hours (D) and in MEF cells with CuONP treatment for 3 hours (F). The effects of $30 \mu$ mol/L p53 stabilizer (nutlin-3 $\alpha$ ) on $\mathrm{CuONP}$-induced viability loss in $\mathrm{HaCaT}(\mathbf{G})$ and $\mathrm{MEF}(\mathbf{H})$ cells with CuONP treatment for 24 hours. The data are presented as the mean \pm standard deviation ( $\mathrm{n}>3$ ).

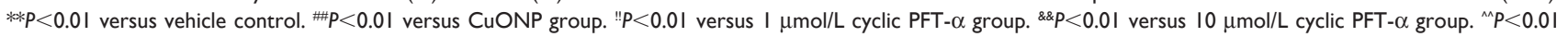
versus nutlin- $3 \alpha$ group.

Abbreviations: CuONP, copper oxide nanoparticles; MEF, mouse embryonic fibroblasts; PFT- $\alpha$, pifithrin- $\alpha$. 

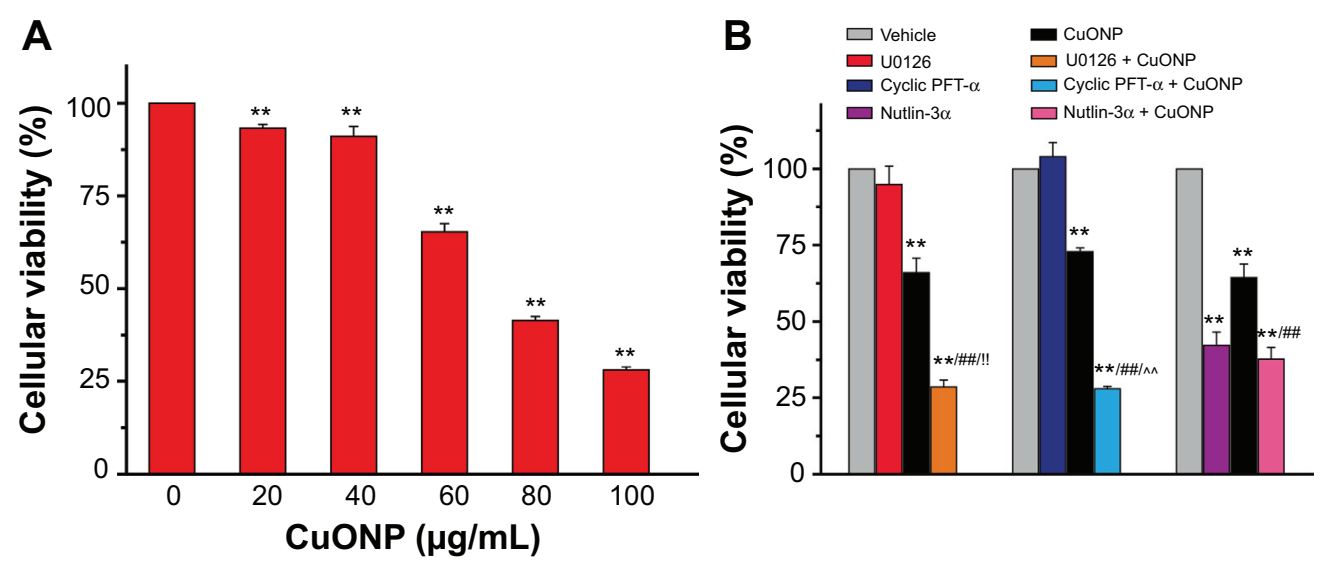

Figure 6 Cytotoxicity of CuONP in primary keratinocytes.

Notes: Viability loss in primary keratinocytes with CuONP treatment for 24 hours (A). The effects of Erk inhibitor (U0I26, $5 \mu$ mol/L), p53 transcriptional inhibitor (cyclic PFT $-\alpha, I \mu \mathrm{mol} / \mathrm{L}$ ), and $\mathrm{p} 53$ stabilizer (nutlin- $3 \alpha, 30 \mu \mathrm{mol} / \mathrm{L}$ ) on viability loss in primary keratinocytes with $60 \mu \mathrm{g} / \mathrm{mL}$ CuONP treatment for 24 hours (B). The data are presented as the mean \pm standard deviation $(n>3)$. ${ }^{* *} P<0.0 \mathrm{I}$ versus vehicle control. ${ }^{\#} P<0.0 \mathrm{I}$ versus CuONP group. " $P<0.0 \mathrm{I}$ versus $\cup 0 \mathrm{I} 26$ group. ${ }^{\wedge} P<0.0 \mathrm{I}$ versus cyclic PFT- $\alpha$ group.

Abbreviations: CuONP, copper oxide nanoparticles; PFT- $\alpha$, pifithrin- $\alpha$.

\section{Discussion}

CuONP have been applied in various industrial and medical areas, such as heat-transfer systems, gas sensors, electronic chips, and biocides. ${ }^{1}$ The skin, respiratory tract, and gastrointestinal tract are the most susceptible to the adverse effects of hazardous materials outside the human body due to their direct exposure to the environment. The skin is the largest among these organs, which increases the risk of being affected. Moreover, potent medical applications utilizing the antibacterial activity of $\mathrm{CuONP}$ give rise to direct contact between $\mathrm{CuONP}$ and the skin. ${ }^{6,7}$ Gauze and bandages containing CuONP could protect wounds against microbial infection. However, there is a risk in using $\mathrm{CuONP}$ application as a microbicide for covering the skin surface: ie, harm to skin-associated cells while killing microorganisms. Keratinocytes and fibroblasts, cooperating to facilitate skin repair, will be affected by $\mathrm{CuONP}$. The redox activities and nanoscale properties of CuONP may promote cytotoxicity to these cells. CuONP exposure has been reported to induce a significant decrease in cellular viability and glutathione levels, and an increase in oxidative stress, apoptosis, and DNA damage in $\mathrm{HaCaT}$ cells. ${ }^{16}$ However, the underlying mechanisms are not well clarified. The present work reveals that cell cycle arrest occurred in CuONP-treated keratinocytes and fibroblasts and that the activation of Erk and $\mathrm{p} 53$ protected both cell types from cytotoxicity.

The proliferation and migration of keratinocytes and fibroblasts are important stages of wound repair and skin development. ${ }^{26} \mathrm{~A}$ loss of skin integrity renders the body vulnerable to invasion by foreign pathogens. Viability loss restrains the proliferation and migration capacity of both cell types, which will undermine cutaneous wound healing and lead to inflammation and infection. Cell cycle progression dysfunction is another pivotal factor affecting proliferation. Generally, one cell divides into two daughter cells when one cell cycle completes, and those progressions are under the control of checkpoints. ${ }^{27}$ Combinations of cyclins and cyclindependent kinases, the main components of checkpoints, vary in different stages of the cell cycle and regulate cell cycle progression. The cell cycle can be blocked in certain stages due to cellular damage and stress signaling, which makes the checkpoints defective. If the protein levels of cyclin A and B do not increase after $\mathrm{G}_{1}$ phase, cell progression cannot pass through $\mathrm{G}_{2} / \mathrm{M}$ phase and complete mitosis. ${ }^{28}$ In the present work, following CuONP treatment, the deregulation of cyclin A and $B$ dynamics led to blockage of $\mathrm{G}_{2} / \mathrm{M}$ transitions and further promoted viability loss (Figures 2 and 3 ).

Erk, JNK, and p38 MAPK compose a large family of MAPKs that regulates various cellular functions and activities, such as proliferation, differentiation, mitosis, survival, and death. ${ }^{29}$ The activation of Erk, p38, and JNK was significantly induced in dose- and time-dependent manners in CuONP-treated HaCaT cells (Figure 4A and B) and MEF cells (Figure $4 \mathrm{C}$ and D). The activation of JNK and p38 MAPK has been reported to promote cell death. ${ }^{30}$ However, neither the inhibition of $\mathrm{p} 38$ activation nor the inhibition of JNK activation prevented viability loss in CuONP-treated $\mathrm{HaCaT}$ and MEF cells (Figure 4G and $\mathrm{H}$ ). These data imply that the activation of $\mathrm{p} 38$ and JNK under CuONP exposure was incidental. Strong Erk activation was induced and sustained for several hours (Figure 4A-D). There are two opposing functions of Erk activation in cell proliferation and cell death. ${ }^{30}$ When Erk activation was suppressed by inhibitors (eg, U0126), cell death due to 
harmful reagents was promoted or suppressed, corresponding to Erk's prosurvival and prodeath functions, respectively. Martin and Pognonec described both functions of Erk in cadmium toxicity, depending on different experimental conditions (eg, the cadmium treatment and cell type). ${ }^{31}$ It was necessary to inhibit Erk activation to uncover the roles that Erk played in CuONP-induced cytotoxicity. The inhibition of Erk activation promoted viability loss in cells treated with both low and high concentrations of $\mathrm{CuONP}$, which implies a prosurvival function for Erk in this context. Several studies reported that CuONP induced reactive oxygen species (ROS) generation in different cells, ${ }^{10,13,18,32}$ including keratinocytes ${ }^{16}$ and fibroblasts. ${ }^{33}$ For example, Alarifi et al reported that CuONP induced dosedependent cell death and ROS generation in keratinocytes. ${ }^{16}$ Considering that ROS generation would activate Erk, ${ }^{29,34,35}$ the dose-dependent Erk activation in the present work might be similarly due to CuONP-induced ROS generation.

p53 is a transcription factor that regulates the expression of hundreds of genes. Those genes are involved in metabolic homeostasis, antioxidant defense, DNA repair, growth arrest, senescence, apoptosis, autophagy, and other processes. The stability of $\mathrm{p} 53$ protein is mainly regulated by interaction with MDM2. The p53/MDM2 complex promotes $\mathrm{p} 53$ degradation by ubiquitylation and blocks the transactivation domain. ${ }^{36}$ Nutlin-3 $\alpha$ can stabilize p53 levels and the transcriptional activity by inhibiting the MDM2-p53 interaction. ${ }^{37}$ The phosphorylation of $\mathrm{p} 53$ at serine 15 stimulates its transactivation. ${ }^{38,39}$ Cyclic PFT- $\alpha$, a cyclic analog of PFT- $\alpha$, inhibits p53dependent gene transcription. ${ }^{40}$ Surprisingly, in the current study, p53 and p-p53 levels decreased in CuONP-treated cells (Figure 5A and B), which implies that p53 did not have a prodeath function. Cyclic PFT- $\alpha$ and nutlin- $3 \alpha$ were used to further evaluate whether $\mathrm{p} 53$ had a prosurvival function. Figure $5 \mathrm{C}-\mathrm{H}$ show that cyclic PFT- $\alpha$ significantly promoted CuONP-induced viability loss in both $\mathrm{HaCaT}$ and MEF cells, whereas nutlin-3 $\alpha$ treatment inhibited the cellular viability loss of CuONP-treated HaCaT cells. These data indicate that p53 is involved in CuONP-induced cytotoxicity and plays a prosurvival role. There was no viability-loss inhibition observed following nutlin-3 $\alpha$ treatment of CuONP-treated MEF cells, which may have resulted from the cytotoxicity of nutlin-3 $\alpha$ to MEF cells. The different responses of HaCaT and MEF cells to nutlin-3 $\alpha$ treatment might have been due to the different p53 statuses of these two types of cells: there are $\mathrm{p} 53$ mutations in $\mathrm{HaCaT}$ cells. ${ }^{41}$ Tovar et al reported that nutlin-3 treatment induced growth inhibition in p53-wildtype fibroblasts. ${ }^{42}$ However, no literature has reported similar phenomena in $\mathrm{HaCaT}$ cells. These reports suggest that $\mathrm{HaCaT}$ cells may be more tolerant to high p53 levels than MEF cells are. Therefore, pretreatment with nutlin-3 $\alpha$ stabilized p53 and increased its levels in MEF cells, resulting in cytotoxicity.

\section{Conclusion}

In conclusion, we have investigated the cytotoxic effects of CuONP on skin-associated HaCaT cells, MEF cells, and primary keratinocytes, as well as the underlying mechanisms. The present study showed that CuONP is toxic to skin-associated cells and that Erk and p53 may be the key factors regulating this cytotoxicity. These results suggest that contact with CuONP may cause damage to the skin and that the activation of Erk and p53 might be a possible way to prevent the potential toxicity of CuONP to the skin.

\section{Acknowledgments}

This work was supported by the National Natural Science Foundation of China $(81071299,81371732)$ and by projects 985 and 211 of Xi' an Jiaotong University and was partially supported by NCET and the Fundamental Research Funds for the Central Universities, and Changjiang Scholars Innovative Research Team in University (PCSIRT:1171).

\section{Disclosure}

The authors report no conflicts of interest in this work.

\section{References}

1. Bondarenko O, Juganson K, Ivask A, Kasemets K, Mortimer M, Kahru A. Toxicity of $\mathrm{Ag}, \mathrm{CuO}$ and $\mathrm{ZnO}$ nanoparticles to selected environmentally relevant test organisms and mammalian cells in vitro: a critical review. Arch Toxicol. 2013;87(7):1181-1200.

2. Ren G, Hu D, Cheng EW, Vargas-Reus MA, Reip P, Allaker RP. Characterisation of copper oxide nanoparticles for antimicrobial applications. Int J Antimicrob Agents. 2009;33(6):587-590.

3. Applerot G, Lellouche J, Lipovsky A, et al. Understanding the antibacterial mechanism of $\mathrm{CuO}$ nanoparticles: revealing the route of induced oxidative stress. Small. 2012;8(21):3326-3337.

4. Hans M, Erbe A, Mathews S, Chen Y, Solioz M, Mucklich F. Role of copper oxides in contact killing of bacteria. Langmuir. 2013;29(52): 16160-16166.

5. Borkow G, Zhou SS, Page T, Gabbay J. A novel anti-influenza copper oxide containing respiratory face mask. PLoS One. 2010;5(6):e11295.

6. Zatcoff RC, Smith MS, Borkow G. Treatment of tinea pedis with socks containing copper-oxide impregnated fibers. Foot (Edinb). 2008; 18(3):136-141.

7. Anita S, Ramachandran T, Rajendran R, Koushik CV, Mahalakshmi M. A Study of the Antimicrobial Property of Encapsulated Copper Oxide Nanoparticles on Cotton Fabric. Textile Research Journal. 2011;81(10): 1081-1088.

8. Perreault F, Popovic R, Dewez D. Different toxicity mechanisms between bare and polymer-coated copper oxide nanoparticles in Lemna gibba. Environ Pollut. 2014;185:219-227.

9. Thit A, Selck H, Bjerregaard HF. Toxicity of $\mathrm{CuO}$ nanoparticles and $\mathrm{Cu}$ ions to tight epithelial cells from Xenopus laevis (A6): effects on proliferation, cell cycle progression and cell death. Toxicol In Vitro. 2013;27(5):1596-1601. 
10. Bulcke F, Thiel K, Dringen R. Uptake and toxicity of copper oxide nanoparticles in cultured primary brain astrocytes. Nanotoxicology. 2014;8(7):775-785.

11. Hanagata N, Zhuang F, Connolly S, Li J, Ogawa N, Xu M. Molecular responses of human lung epithelial cells to the toxicity of copper oxide nanoparticles inferred from whole genome expression analysis. ACS Nano. 2011;5(12):9326-9338.

12. Sun T, Yan Y, Zhao Y, Guo F, Jiang C. Copper oxide nanoparticles induce autophagic cell death in A549 cells. PLoS One. 2012;7(8):e43442.

13. Piret JP, Jacques D, Audinot JN, et al. Copper(II) oxide nanoparticles penetrate into HepG2 cells, exert cytotoxicity via oxidative stress and induce pro-inflammatory response. Nanoscale. 2012;4(22): $7168-7184$

14. Cuillel $\mathrm{M}$, Chevallet $\mathrm{M}$, Charbonnier $\mathrm{P}$, et al. Interference of $\mathrm{CuO}$ nanoparticles with metal homeostasis in hepatocytes under sub-toxic conditions. Nanoscale. 2014;6(3):1707-1715.

15. Xu J, Li Z, Xu P, Xiao L, Yang Z. Nanosized copper oxide induces apoptosis through oxidative stress in podocytes. Arch Toxicol. 2013;87(6): 1067-1073.

16. Alarifi S, Ali D, Verma A, Alakhtani S, Ali BA. Cytotoxicity and genotoxicity of copper oxide nanoparticles in human skin keratinocytes cells. Int J Toxicol. 2013;32(4):296-307.

17. Karlsson HL, Cronholm P, Gustafsson J, Möller L. Copper oxide nanoparticles are highly toxic: a comparison between metal oxide nanoparticles and carbon nanotubes. Chem Res Toxicol. 2008;21(9): 1726-1732.

18. Ahamed M, Siddiqui MA, Akhtar MJ, Ahmad I, Pant AB, Alhadlaq HA. Genotoxic potential of copper oxide nanoparticles in human lung epithelial cells. Biochem Biophys Res Commun. 2010;396(2):578-583.

19. Cronholm P, Karlsson HL, Hedberg J, et al. Intracellular uptake and toxicity of $\mathrm{Ag}$ and $\mathrm{CuO}$ nanoparticles: a comparison between nanoparticles and their corresponding metal ions. Small. 2013;9(7):970-982.

20. Semisch A, Ohle J, Witt B, Hartwig A. Cytotoxicity and genotoxicity of nano - and microparticulate copper oxide: role of solubility and intracellular bioavailability. Part Fibre Toxicol. 2014;11:10.

21. Zhu J, Li D, Chen H, Yang X, Lu L, Wang X. Highly dispersed CuO nanoparticles prepared by a novel quick-precipitation method. Mater Lett. 2004;58(26):3324-3327.

22. Mosmann T. Rapid colorimetric assay for cellular growth and survival: application to proliferation and cytotoxicity assays. J Immunol Methods. 1983;65(1-2):55-63.

23. Liang CC, Park AY, Guan JL. In vitro scratch assay: a convenient and inexpensive method for analysis of cell migration in vitro. Nat Protoc. 2007;2(2):329-333.

24. Darzynkiewicz Z, Juan G, Bedner E. Determining cell cycle stages by flow cytometry. Curr Protoc Cell Biol. 2001; Chapter 8:Unit 8.4.

25. Albarenque SM, Doi K. T-2 toxin-induced apoptosis in rat keratinocyte primary cultures. Exp Mol Pathol. 2005;78(2):144-149.

26. Bielefeld KA, Amini-Nik S, Alman BA. Cutaneous wound healing: recruiting developmental pathways for regeneration. Cell Mol Life Sci. 2013;70(12):2059-2081.
27. Pietenpol JA, Stewart ZA. Cell cycle checkpoint signaling: cell cycle arrest versus apoptosis. Toxicology. 2002;181-182:475-481.

28. Duronio RJ, Xiong Y. Signaling pathways that control cell proliferation. Cold Spring Harb Perspect Biol. 2013;5(3):a008904.

29. $\mathrm{Lu} \mathrm{Z}, \mathrm{Xu} \mathrm{S}$. ERK1/2 MAP kinases in cell survival and apoptosis. IUBMB Life. 2006;58(11):621-631.

30. Subramaniam S, Unsicker K. Extracellular signal-regulated kinase as an inducer of non-apoptotic neuronal death. Neuroscience. 2006;138(4): 1055-1065.

31. Martin P, Pognonec P. ERK and cell death: cadmium toxicity, sustained ERK activation and cell death. FEBS J. 2010;277(1):39-46.

32. Siddiqui MA, Alhadlaq HA, Ahmad J, Al-Khedhairy AA, Musarrat J, Ahamed M. Copper oxide nanoparticles induced mitochondria mediated apoptosis in human hepatocarcinoma cells. PLoS One. 2013; 8(8):e69534.

33. Akhtar MJ, Ahamed M, Fareed M, Alrokayan SA, Kumar S. Protective effect of sulphoraphane against oxidative stress mediated toxicity induced by $\mathrm{CuO}$ nanoparticles in mouse embryonic fibroblasts BALB 3T3. J Toxicol Sci. 2012;37(1):139-148.

34. Keshari RS, Verma A, Barthwal MK, Dikshit M. Reactive oxygen species-induced activation of ERK and p38 MAPK mediates PMAinduced NETs release from human neutrophils. J Cell Biochem. 2013; 114(3):532-540.

35. Ortuño-Sahagún D1, González RM, Verdaguer E, et al. Glutamate excitotoxicity activates the MAPK/ERK signaling pathway and induces the survival of rat hippocampal neurons in vivo. J Mol Neurosci. 2014; 52(3):366-377.

36. Levine AJ, Oren $\mathrm{M}$. The first 30 years of $\mathrm{p} 53$ : growing ever more complex. Nat Rev Cancer. 2009;9(10):749-758.

37. Vassilev LT, Vu BT, Graves B, et al. In vivo activation of the p53 pathway by small-molecule antagonists of MDM2. Science. 2004; 303(5659):844-848.

38. Faour WH, He Q, Mancini A, Jovanovic D, Antoniou J, Di Battista JA. Prostaglandin E2 stimulates p53 transactivational activity through specific serine 15 phosphorylation in human synovial fibroblasts. Role in suppression of c/EBP/NF-kappaB-mediated MEKK1-induced MMP-1 expression. J Biol Chem. 2006;281(29):19849-19860.

39. Dumaz N, Meek DW. Serine 15 phosphorylation stimulates p53 transactivation but does not directly influence interaction with HDM2. EMBO J. 1999;18(24):7002-7010.

40. Barchéchath SD, Tawatao RI, Corr M, Carson DA, Cottam HB. Inhibitors of apoptosis in lymphocytes: synthesis and biological evaluation of compounds related to pifithrin-alpha. J Med Chem. 2005;48(20): 6409-6422.

41. Lehman TA, Modali R, Boukamp P, et al. p53 mutations in human immortalized epithelial cell lines. Carcinogenesis. 1993;14(5):833-839.

42. Tovar C, Rosinski J, Filipovic Z, et al. Small-molecule MDM2 antagonists reveal aberrant p53 signaling in cancer: implications for therapy. Proc Natl Acad Sci USA. 2006;103(6):1888-1893.
International Journal of Nanomedicine

\section{Publish your work in this journal}

The International Journal of Nanomedicine is an international, peerreviewed journal focusing on the application of nanotechnology in diagnostics, therapeutics, and drug delivery systems throughout the biomedical field. This journal is indexed on PubMed Central, MedLine, CAS, SciSearch $\AA$, Current Contents $\AA /$ Clinical Medicine,

\section{Dovepress}

Journal Citation Reports/Science Edition, EMBase, Scopus and the Elsevier Bibliographic databases. The manuscript management system is completely online and includes a very quick and fair peer-review system, which is all easy to use. Visit http://www.dovepress.com/ testimonials.php to read real quotes from published authors. 